\section{Open Peer Commentary}

\section{Beware of individual differences}

\author{
Peter Borkenau and Nadine Mauer \\ Department of Psychology, Martin-Luther University, 06099 Halle, Germany. \\ p.borkenau@psych.uni-halle.de n.mauer@psych.uni-halle.de \\ http://www.psych.uni-halle.de/borkenau/borkenae.htm \\ http://www.psych.uni-halle.de/nadine/mauere.htm
}

\begin{abstract}
Most judgmental biases are found at the level of samples, but do not apply to each person; they reflect prevailing, but not universal, response tendencies. We suggest that it is more promising to study differences between biased and unbiased persons, and between easier and more difficult tasks, than to generalize from a majority of research participants to humans in general.
\end{abstract}

That humans err is hardly new. The ancient Romans said errare humanum est. The intriguing issue in research on judgmental biases is, therefore, not that humans may err in many ways, but to understand why human reasoning that results in adaptive behavior under most circumstances sometimes goes astray. We agree with Krueger \& Funder (henceforth $\mathrm{K} \& \mathrm{~F}$ ) that this perspective has been lost in research on judgmental biases, and we suggest that neglect of individual differences constitutes part of the problem.

Research on judgmental biases yields main effects and individual differences. Usually, a majority of the respondents shows the "human" bias, whereas a minority shows the opposite bias or no bias at all. For example, Tversky and Kahneman (1974) observed that the majority, but not all, of their respondents neglected base rates and sample sizes. Moreover, variations in the framing of a problem may affect the error rate (Hertwig \& Gigerenzer 1999). This shows that respondents vary in relevant knowledge and in cognitive ability, and that tasks vary in difficulty. Although it is productive to study the hierarchy of the difficulty of such problems, as well as which errors covary across respondents, to know whether or not more than $50 \%$ of the research participants exhibit a particular response tendency is unlikely to result in major insights.

Unfortunately, the individual differences are usually masked by the way the data are analyzed: as sample means, followed by comparisons of the observed mean to expectations under an elaborated normative model. If a discrepancy is obtained, it is claimed that a "human" bias has been discovered. Ross (1977) even suggested an intuitive psychologist as a personification of such tendencies at the aggregate level. But what about those research participants who were not susceptible to the bias under study? Are they rational psychologists? And why did they respond appropriately? In many experiments, they probably had better access to relevant knowledge, they understood the instructions as meant by the experimenter, or their affect-related schemata did not bias their judgments. Obviously, to suggest such answers is not as spectacular as to discover a "human" or even a "fundamental human" bias, but it reveals more about human cognitive processes and about the sources of both accurate and inaccurate judgments.

Krueger and Funder noticed that some tasks that were used to study judgmental biases might qualify as items in an intelligence test. We agree, but would like to add that other biases are related to long-term affect. That depressives tend to be sadder but wiser (Taylor \& Brown 1988) is a case in point. Another example is the tendency to report above-average levels in desirable attributes (Klar \& Giladi 1997). Note that this tendency does not apply to each individual; whereas a majority of the respondents claims to be above average, a minority reports to be below average.

We are going to illustrate this with some data on the so-called optimistic bias, which is a tendency to estimate one's personal risk to experience aversive events as being lower, and one's chances to experience pleasant events as being higher, than those of the average person of one's age, sex, and education (Helweg-Larsen \& Shepperd 2001). We let 114 students (71 women and 43 men) estimate the likelihood (in percentages) that: (a) they would experience 14 pleasant events (e.g., to be successful in their job), (b) another person of their age, sex, and education would experience these pleasant events, (c) they would experience 18 aversive events (e.g., to die in a traffic accident), and (d) another person of their age, sex, and education would experience these aversive events. To obtain measures of bias, difference scores were computed by subtracting estimates for other persons from estimates for oneself. Moreover, the risk estimates and difference scores were separately averaged across the 14 pleasant and the 18 aversive events.

Consistent with the optimistic bias view, the respondents estimated the chances that the 14 pleasant events would occur to themselves $(M=57.02, S D=11.39)$ as higher than that they would occur to another person $(M=49.30, S D=11.29) ; t$ (113) $=6.72, p<.001$. Correspondingly, they estimated the chances that the 18 aversive events would occur to themselves $(M=21.21$, $S D=12.55)$ as lower than that they would occur to another person $(M=24.51, S D=12.75) ; t(113)=3.19, p<.01$. That, however, is only half the story: A minority of $21.9 \%$ of the respondents indicated that pleasant events were less likely to occur to themselves than to others, and $31.6 \%$ indicated that aversive events were more likely to occur to themselves than to others. Thus, a substantial minority of the respondents showed a pessimistic bias. To check whether the individual differences in judgmental tendencies were consistent across particular events, we estimated the internal consistencies of the difference scores and obtained alphas of .67 and .83 for pleasant and aversive events, respectively. Thus, the individual differences were reliable.

Moreover, when the estimated risks for oneself were compared to the actual risks, instead of the risks estimated for others, the majority of the respondents overestimated some risks. For example, the average risk estimate to die in a traffic accident was $16.05 \%$ for oneself and $17.15 \%$ for another person. But with a population in Germany of more than 80 million, with about 8,000 persons dying in traffic accidents each year, and a remaining life expectancy of our participants of approximately 55 years, their actual risk to die in a traffic accident was less than $1 \%$. Risk estimates of $0 \%$ or $1 \%$ were provided by $26.3 \%$ of the respondents only. Thus, when actual risk was used as the standard of comparison, $73.7 \%$ of the respondents overestimated their risk.

There are two implications of these findings for research on judgmental biases. First, like many other biases, the "optimistic bias" does not apply to all humans; rather, it reflects that there are more persons who show one sort of judgmental tendency than there are persons who show the opposite sort. Second, depending on the particular standards to which the actual judgments are compared, opposite judgmental biases can be shown.

\section{Functional clothes for the emperor}

\section{Gary L. Brase \\ Department of Psychological Sciences, University of Missouri-Columbia, Columbia, MO 65211. BraseG@missouri.edu}

\begin{abstract}
A more complete and balanced theoretical framework for social psychology, as recommended in the target article, must include functional explanations of processes - moving beyond enumerations of processes and their properties. These functional explanations are at a different, but complementary, level from process descriptions. The further advancement of social psychology relies on the incorporation of such multilevel explanations.
\end{abstract}

Krueger \& Funder $(\mathrm{K} \& \mathrm{~F})$ state that "the problem-seeking approach [in social psychology] tends to be atheoretical" (target ar- 
ticle, sect. 3). This claim may be met with some incredulous denials; there are, after all, a cornucopia of theories in social psychology, many of which are discussed by $K \& F$ themselves. The theoretical vacuum truly does exist, however, and it resides in the need for functional explanations of processes, rather than mere enumeration of theoretical processes and properties. Functional explanations (e.g., what is phenomenon $\mathrm{X}$ designed to do?) are at a different explanatory level from process descriptions. In the information-processing model described by Marr (1982), functional explanations are at the computational level, whereas process explanations are at the algorithmic/representational level. Therefore, the further advancement of social psychology relies not only on the interaction of situational and dispositional factors, as $\mathrm{K} \& \mathrm{~F}$ illustrate very well, but also on multilevel explanations.

Thus, in a very real sense, $\mathrm{K} \& \mathrm{~F}$ are correct in pointing out that social psychology has little in the way of solid theoretical clothes with which to dress their findings. This brings up, even more urgently, the issue of what could constitute appropriate "clothes" for the discipline. Like pointing out that the emperor has no clothes, it can be difficult to acknowledge that something does not exist when many other people have a stake in saying that it does exist. The dilemma is not just stating that social psychology lacks a metatheoretical framework, but having this message come from someone who is above dismissal as one "unfit for his office or too stupid for any use" (in the words of Hans Christian Andersen [1916/1995]). K\&F will hopefully be recognized for the credentials they do, in fact, possess.

A further problem, once the theoretical imbalance and shortcomings in social psychology are acknowledged, is to develop a proper theoretical framework for the functional abilities that constitute social psychology. K\&F suggest a couple directions for this work: the functional considerations derived from theories of bounded rationality and evolutionary psychology. But primarily, $K \& F$ have chosen to focus on the initial issue of the adequacy of theory construction in social psychology in terms of both internal and external consistency, and they have some excellent suggestions for methodological tools to improve social psychological research. They devote only a short section to the issue of how social psychology should proceed in developing a better framework for understanding the functional design of the mind. This commentary elaborates on this topic for a simple reason: With the realization that social psychology - like the fabled emperor - has no functional clothes, there is an acute need for clothes.

The emperor of the children's fable shows himself in the end to be a fool who cannot acknowledge his error, and is therefore held up for further ridicule. Let us not make the same mistake in social psychology, particularly when proper metatheoretical clothes are so close at hand. With the implementation of an evolutionary theoretical paradigm, social psychology phenomena become amenable to interactive and integrated functional explanations that cover the range of relevant behaviors. This is true not just for the brief examples given in the target article (i.e., the representativeness heuristic, the availability heuristic, consensus bias, selfenhancement, and the fundamental attribution error), but also for other aspects of social behavior and cognition that K\&F noted.

For example, the study of helping behaviors in social psychology has an obvious parallel in the study of altruism in evolutionary biology. These parallels are strong and informative, despite the differences in initial orientation (violations of helping norms in social psychology; the development of any helping in biology) and behaviors typically targeted (emergency helping of strangers in social psychology; kin-based and repeated interaction helping in biology). Recent work to integrate these disciplines have produced strong support for their compatibility and have begun to show the way for an integrated overall theory of altruism/helping (e.g., Burnstein et al.; Cialdini et al. 1997). As emphasized in the target article, this integration and resulting functional theory produces an explanatory framework that covers the complete range of behaviors (i.e., all degrees of helping, from the life-threatening, to the mundane, to failures to help).
Another example is the evolutionary analysis of the fundamental attribution error (FAE; Andrews 2001), which has provided a functional-level description of attributional processes that can explain the historical adaptiveness of the FAE, its current maladaptive qualities, and predicts further functional design features of the FAE that are likely to exist based on this account. In addition to being consistent with existing knowledge of the evolutionary history of the human species, this description is also boundedly rational in that it specifies the conceptual reference class of conditions under which the FAE will be adaptive and accurate (and thereby the conditions outside those bounds as well).

Beyond the benefits of metatheoretical clothes for the existing body of research in social psychology, there are further benefits to adopting a functional-level framework for social behavior and cognition. Such a framework allows social psychology to become better integrated with other behavioral sciences, which will facilitate scientific progression (Tooby \& Cosmides 1992). A more direct benefit to researchers is that a functional framework can facilitate the identification of rich topics and hypotheses that are both important and, heretofore, little researched. Daly et al. (1997), for example, point out that the topic of kinship - studied extensively in anthropology, biology, and sociology - is nearly absent as an important topic in social psychology.

$\mathrm{K} \& \mathrm{~F}$ allude to, but do not emphasize, that bounded rationality is consonant with evolutionary psychology. In fact, one aspect of the ecological rationality program of Gigerenzer and colleagues is that it is a specific application of evolutionary insights into the fields of judgments under uncertainty and decision-making (Gigerenzer \& Todd 1999). Such a theory of evolved functional design, by virtue of its emphasis on what cognitive mechanisms are designed to solve (and the subsequent ability to place failures of cognitive mechanisms into context), yields an account that covers a range of behavioral and cognitive performances. For example, the theory that the mind is predisposed to register numerical information in natural frequency formats (Gigerenzer \& Hoffrage 1995), not only provides an explanation for certain patterns of judgments under uncertainty, but also explains some of the difficulties children have in the course of mathematics instruction (Brase 2002a). This has led to further specifications about the nature of information representation in the mind (e.g., Brase 2002b; Brase et al. 1998).

\section{ACKNOWLEDGMENTS}

The author thanks David Over and Sandra Brase for helpful comments and advice in the development of this comment.

\section{Additional requirements for a balanced social psychology}

\section{Siu L. Chow}

Department of Psychology, University of Regina, Regina, Saskatchewan S4S OA2, Canada.Siu.Chow@uregina.ca http://uregina.ca/chowsI

Abstract: Ambiguous data obtained by deception say nothing about social behavior. A balanced social psychology requires separating statistical hypotheses from substantive hypotheses. Neither statistical norms nor moral rules are psychological theories. Explanatory substantive theories stipulate the structures and processes underlying behavior. The Bayesian approach is incompatible with the requirement that all to-be-tested theories be given the benefit of the doubt.

One may agree with Krueger \& Funder's (K\&F's) global thesis that social psychology depicts too negative a picture of human nature. However, they concede too much to the "negative psychology" camp. For example, they could have noted that Milgram's (1963) data were rendered ambiguous by the deception paradigm used. The subjects that were told to facilitate the learner-confederate's learning with electric shocks were confronted with two in- 